\title{
Muscle activity response of the audience during an experimental music performance
}

\author{
Victor Gonzalez Sanchez \\ RITMO Centre for Interdisciplinary \\ Studies in Rhythm, Time and Motion \\ Department of Musicology, \\ University of Oslo \\ v.e.g.sanchez@imv.uio.no
}

\author{
Agata Żelechowska \\ RITMO Centre for Interdisciplinary \\ Studies in Rhythm, Time and Motion \\ Department of Musicology, \\ University of Oslo \\ agata.zelechowska@imv.uio.no
}

\author{
Alexander Refsum Jensenius \\ RITMO Centre for Interdisciplinary \\ Studies in Rhythm, Time and Motion \\ Department of Musicology, \\ University of Oslo \\ a.r.jensenius@imv.uio.no
}

\begin{abstract}
This exploratory study investigates muscular activity characteristics of a group of audience members during an experimental music performance. The study was designed to be as ecologically valid as possible, collecting data in a concert venue and making use of lowinvasive measurement techniques. Muscle activity (EMG) from the forearms of 8 participants revealed that sitting in a group could be an indication of a level of group engagement, while comparatively greater muscular activity from a participant sitting at close distance to the stage suggests performance-induced bodily responses. The self-reported measures rendered little evidence supporting the links between muscular activity and live music exposure, although a larger sample size and a wider range of music styles need to be included in future studies to provide conclusive results.
\end{abstract}

\section{CCS CONCEPTS}

- Applied computing $\rightarrow$ Performing arts; Sound and music computing; Engineering;

\section{KEYWORDS}

Music, Movement, EMG, Performance, Audience, Interaction

\section{INTRODUCTION}

A number of studies have looked at different types of physiological and psychological effects of music on the human body and its motion $[6,7,11,12,18]$. The documented bodily responses to music range from observable motion like finger tapping [10], head nodding [16] or whole body sway [3], to internal reactions such as changes in heart rate, breathing, and skin conductance [1, 4]. Although studies investigating the effects of music listening on muscular activity are still scarce, there is evidence of such a link, with Blood and Zatorre observing that pleasant responses to subjectselected music induced significant changes in muscle activity, measured through a polygraph instrumentation system [1].

Since body motion is intrinsically related to muscle activity, characterization of small, and sometimes involuntary, motion can lead to insight into muscular tension. Jensenius et al. [9] have characterized the musical influence on groups of people that try to stand still, and found that participants exhibited a significant increase in motion while being exposed to music.

Such music-induced (and involuntary) motion has been found to be partially attributed to the acoustic sensitivity of the vestibular system. Todd [19] observed that acoustic sequences with varying energy, amplitude, or pulse, produce vestibular response signals

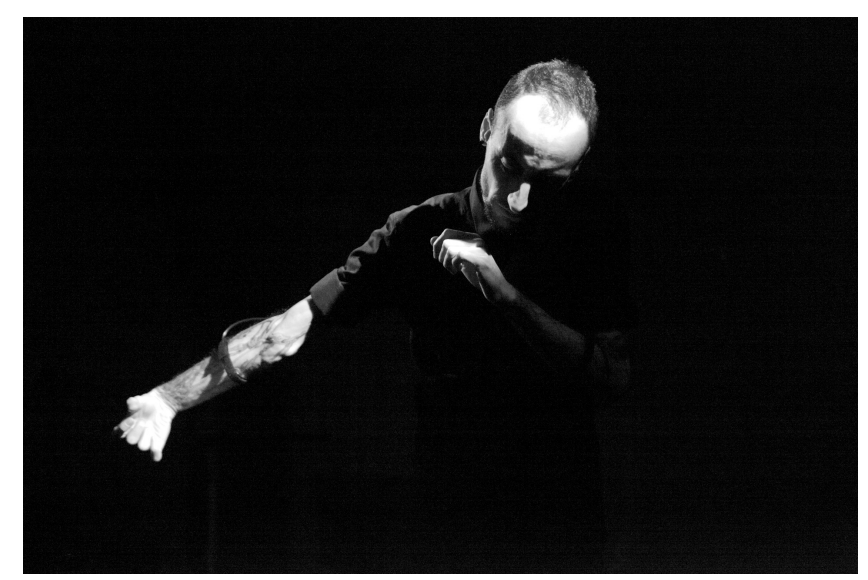

Figure 1: Marco Donnarumma performs with his XTH sensors at the concert we have studied (Photo: Simen Kjellin).

and a modulated sense of motion. Furthermore, the vestibular system, and in particular the lateral vestibulo-spinal tract, has been found to influence motor control through the spinal motor neurons, generating muscle activity responses after being exposed to sound stimuli [2, 20, 21]. Sheykholeslami et al. [17], for example, observed activity bursts from the sternocleidomastoid muscle as response to bone-conducted tone stimuli.

Most of the above mentioned studies on music- and soundinduced motion and muscle activity have been conducted in laboratories and making use of synthetic and prerecorded stimuli. Such a setting provides greater control over the conditions and measurement techniques, but inevitably affects the ecological validity of the studies. Moreover, although music-induced motion and physiological changes have been explored in performers [13, 15], studies on the physiological effects of music on the audience are still scarce.

In this study, we collected electromyography (EMG) and questionnaire data from a concert audience before and during a live music performance. The aim was to identify traces of music-evoked muscle activity as well as assessing the level of performance- and group-induced engagement. Further understanding of the responses of physiological traits to musical stimuli can provide developers and performers with fundamental insight into human immersive experience. Moreover, the physiological and biological implications of such responses can lead to the development of applications in the clinical field. 


\section{METHODOLOGY}

\subsection{Live Performance and Participants}

EMG data was collected from members of the audience attending MusicLab, an art-science outreach event at the University of Oslo. Ten audience members volunteered to participate in the study, and were instrumented with one wireless EMG sensor on the dominant forearm before the event started. Data from two of the participants was not processed due to wireless connectivity issues during the live performance, thus reducing the data set to $8(4$ male, mean age $=$ 37.8 years, $\mathrm{SD}=16.8$ and 4 female, mean age $=39.5$ years, $\mathrm{SD}=18.4$ ) The participants volunteered from an audience of approximately 80 people and the event was held at in a student bar/club-venue at the University of Oslo.

The evening program consisted of a 30-minute panel discussion, and a set of performances. The main performance, by Marco Donnarumma,${ }^{1}$ consisted of two experimental music pieces played with the XTH Sense [5], a biophysical instrument developed by the performer (Figure 1). The first piece, Ominous, was 9 minutes long, while the second piece, Music for flesh II, was 13 minutes long. Both pieces were based on the augmented sound of the performer's muscles, and the performance consisted of choreographed hand and arm movements.

\subsection{Data Acquisition}

The participants were informed of the format of the experiment and were asked to behave naturally during the event. One Delsys Trigno (Boston, MA) wireless EMG electrode was placed on the flexor carpi radialis location on the ventral aspect of the forearm, based on suggestions from SENIAM. ${ }^{2}$ EMG signals were recorded at $2000 \mathrm{~Hz}$ using the Delsys EMGWorks software during the last 5 minutes of the introductory panel and during both pieces of the main performance. The data collected during the panel discussion was used as EMG Baseline (BLEMG) data.

After the performance the participants were asked to complete a small set of questionnaires in order to gather data on the demographics and music related experiences, and to evaluate subjective involvement in the presented music: the Barcelona Music Reward Questionnaire (BMRQ) [14], the Beat Alignment Test questionnaire (BAT) [8], and a demographics survey. The BMRQ questionnaire was used to investigate the features of music experience that could explain the range of factors associated in music experience reward [14], while the BAT questionnaire was aimed at assessing features such as self-reported sense of rhythm or physical coordination, and learning details about potential musical training of the participant [14]. Answers to both questionnaires were processed according to their respective guidelines.

\subsection{Data Analysis}

Maximum root mean square values from the recorded EMG data were computed (RMS window length $=0.125 \mathrm{~s}$, RMS window overlap $=0.0625 \mathrm{~s})$ in order to normalize against baseline EMG (BLEMG) data collected during the panel discussion ('no music' condition).

\footnotetext{
${ }^{1} \mathrm{http}: / /$ marcodonnarumma.com

${ }^{2}$ http://www.seniam.org
}

The resulting normalized EMG (NEMG) is expressed as a percentage of the BLEMG, thus reducing user-dependent variance in the data and increasing the reliability of between-subjects comparisons (Figure 2).
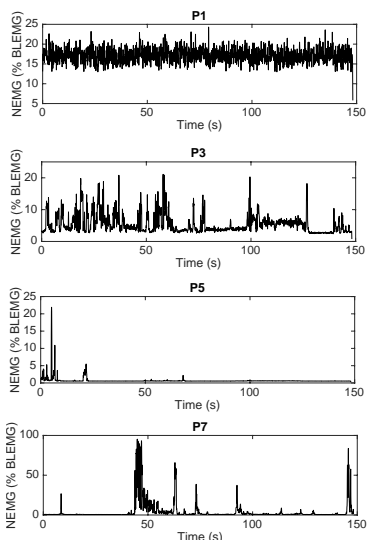
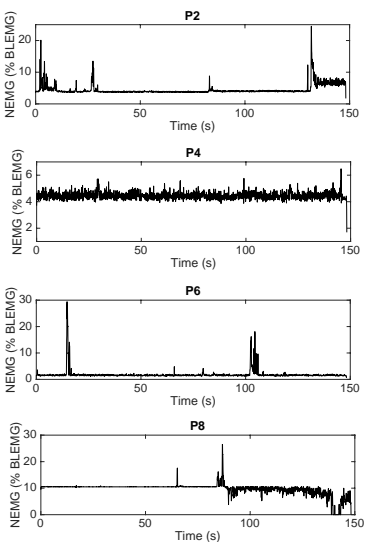

Figure 2: Normalized EMG from all 8 recorded participants during the first segment (150s) of piece 1, Ominous.

Differences in mean NEMG between participants and between two segments from each performed piece were assessed by oneway analysis of variance (ANOVA). Post-hoc pair-wise comparisons were examined through the Tukey multiple comparison method. A Pearson correlation was used to assess links between results from the questionnaires and the mean NEMG across participants.

\section{RESULTS}

\subsection{EMG comparison between participants}

The EMG data was split into two segments for both pieces in order to assess changes in audience response across different segments of the performed pieces, while at the same time reducing the size of the data series for analysis. One-way ANOVA between participants showed statistically significant differences in NEMG across pieces and segments at the 0.05 level $(\mathrm{p}<0.001)$. Post-hoc Tukey multiple comparisons showed no statistically differences in mean NEMG between participants 2 and 4 during segment 1 of piece 1 , while mean NEMG from participants 7 and 3 approached the confidence interval, indicating a degree of similarity (Figure 3). During the second segment of piece 1, participants 2 and 7 had no statistically significant differences in mean NEMG, while activity from participants 3 and 4 approached the confidence interval for no differences (Figure 3). Differences in mean NEMG during the first segment of the second piece, from participants 3 and 4 were found not significant (Figure 4), while mean NEMG from participants 2, 4 and 6 were statistically similar during the second segment (Figure 4).

\subsection{Analysis of questionnaires and EMG}

Scores from the BMRQ and BAT questionnaires (Table 1) were correlated with mean NEMG across participants to quantify the links between muscle activity and the experience of reward in music and the beat perception ability. Pearson correlation between mean NEMG and the features Emotional Evocation (EE), Sensory-Motor 

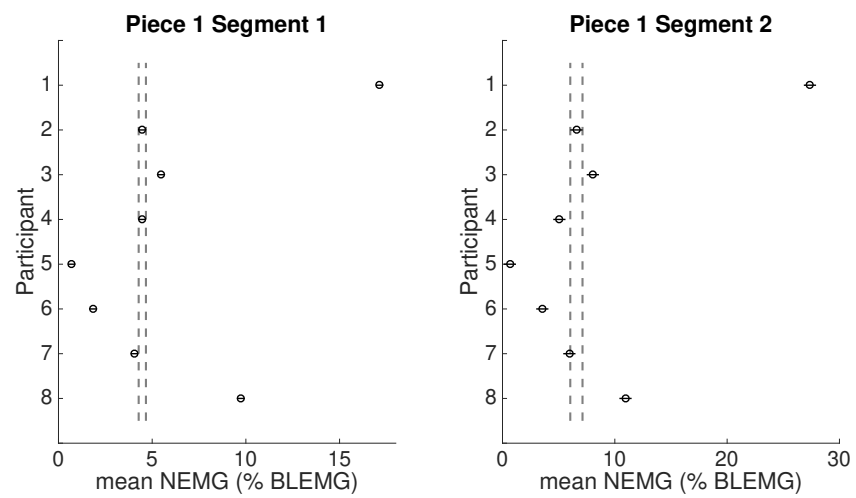

Figure 3: Mean NEMG from all 8 participants with confidence interval for statistically significant differences from Tukey post-hoc test.
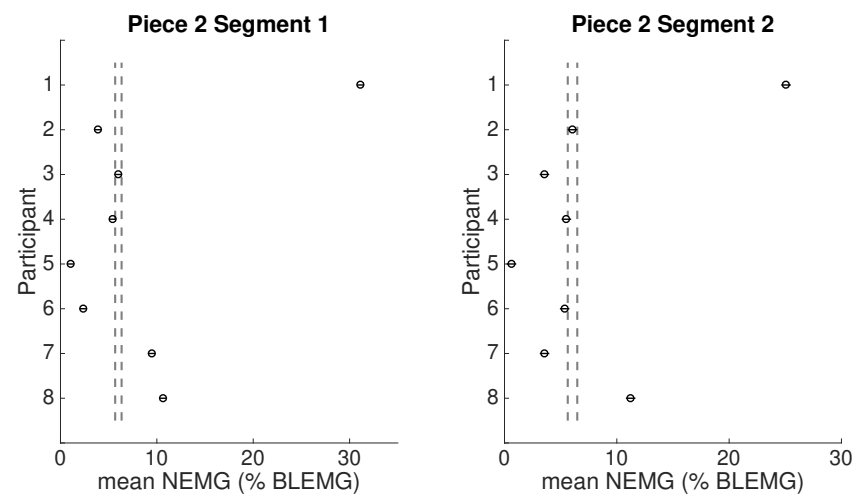

Figure 4: Mean NEMG from all 8 participants with confidence interval for statistically significant differences from Tukey post-hoc test.

(SM), Mood Regulation (MR), Musical Seeking (MS) and Social Reward (SR) factors scored from the BMRQ were not statistically significant across participants for all the segments and pieces ( $\mathrm{p}$ $>0.05$ ). However, negative correlation between the SM factor and NEMG approached significance in both segments from the first piece $(\mathrm{p}=0.095, \mathrm{r}=-0.62$ for segment 1 , and $\mathrm{p}=0.065, \mathrm{r}=-0.67$ for segment 2) suggesting a moderate link between muscular activity and self-reported sensory-motor experience to music.

Scores of how often the participants listen to music, dance, and perform physical activities from the BAT questionnaire were not significantly correlated with mean NEMG across participants for all segments and pieces ( $p>0.05)$. Correlation between NEMG and the self-reported sense of rhythm, physical coordination, and age also failed to reach significance at the same 0.05 level.

\section{DISCUSSION}

Arm muscular activity, measured as baseline-normalized EMG, was shown to differ significantly across participants (one-way ANOVA, $p>0.05)$. This is as expected, since the participants were recorded for a fairly long period of time, and they were not instructed to sit
Table 1: Scores from the BMRQ and BAT questionnaires across participants. BMRQ: Emotional Evocation (EE), Sensory-Motor (SM), Mood Regulation (MR), Musical Seeking (MS), and Social Reward (SR). BAT: Music Listening Frequency (MLF), Dancing Frequency (DF), Sense of Rhythm (SRy), Frequency of Physical Activities (FPA), and Physical Coordination (PC)

\begin{tabular}{|l|lllll|lllll|}
\hline \multirow{2}{*}{ Participant } & \multicolumn{7}{|c|}{ BMRQ } & \multicolumn{5}{c|}{ BAT } \\
& EE & SM & MR & MS & SR & MLF & DF & SRy & FPA & PC \\
\hline 1 & 16 & 13 & 19 & 13 & 19 & 4 & 3 & 4 & 5 & 4 \\
2 & 18 & 15 & 16 & 15 & 18 & 4 & 4 & 5 & 5 & 5 \\
3 & 16 & 13 & 18 & 15 & 18 & 4 & 4 & 5 & 4 & 4 \\
4 & 16 & 14 & 17 & 11 & 12 & 5 & 2 & 3 & 4 & 3 \\
5 & 17 & 16 & 19 & 18 & 19 & 5 & 5 & 5 & 5 & 5 \\
6 & 20 & 17 & 20 & 16 & 19 & 5 & 3 & 5 & 5 & 5 \\
7 & 16 & 14 & 18 & 12 & 17 & 5 & 2 & 3 & 2 & 3 \\
8 & 15 & 14 & 13 & 11 & 16 & 5 & 3 & 4 & 2 & 4 \\
\hline Mean & 16.8 & 14.5 & 17.5 & 13.9 & 17.3 & 4.6 & 3.3 & 4.3 & 4 & 4.1 \\
S.D. & 1.6 & 1.4 & 2.2 & 2.5 & 2.4 & 0.5 & 1.0 & 0.9 & 1.3 & 0.8 \\
\hline
\end{tabular}

as still as possible. The muscular activity can therefore be interpreted as differences in both engagement and behaviour between audience members. Tukey post-hoc pair-wise comparisons, however, showed similarities between a smaller group of participants, with mean NEMG from participants 2, 3, 4 and 7 near or within confidence level for similarities across the performance's pieces and segments (Figure 3). Participant 1 had the largest mean NEMG across pieces and segments, with large differences when compared against the rest of the group. Moreover, visual inspection of video footage showed higher levels of engagement from participant 1 . Interestingly, the position of the participants in the venue during the performance seems to be related with these results. Participants $2,3,7$, and 4 were sitting close to each other, while participant 1 was located near the stage with no instrumented volunteers nearby (Figure 5). Data from participants 5 and 6 presents larger variability, with no clear pattern revealed by the post-hoc test.

When comparing responses to the BMRQ and BAT with NEMG, correlation coefficients were low and lacked statistical significance across pieces and segments ( $p>0.05)$. Factors from the BMRQ measuring feelings of reward and pleasure to music have been shown to correlate negatively with age and positively with subjective aesthetics [14], although there is still a lack of evidence of the links between such BMRQ features and quantitative physiological factors. The lack of correlation between the self-reported ability to perceive a beat as measured by the BAT and NEMG can be attributed to the characteristics of the music performed, which primarily consisted of drones, and with no regular pulse.

\section{CONCLUSIONS}

In this article, we aimed at characterizing EMG data from an audience during an experimental music live performance in order to identify patterns of music-induced and group-induced changes in muscular activity. The study was designed to enhance the ecological validity of the results, collecting data in a concert venue and making use of low-invasive measuring techniques. Normalized EMG similarities between participants sitting in a group could be an indication of a level of group engagement, while comparatively 
Stage
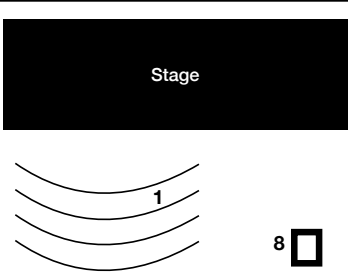

$8 \square$

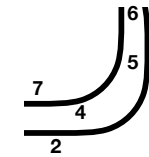

3

Figure 5: Diagram showing the approximate position of the participants in the concert venue. Participant \#1 was sitting on a chair in front of the stage, \#8 at a table, and the rest in the amphi seating further back.

greater muscular activity from a participant sitting at close distance to the stage suggests performance-induced bodily responses. The use of BAT and BMRQ as self-reported measures of music experience and beat perception rendered little evidence supporting the links between muscular activity and live music exposure, although a larger sample size and a wider range of music styles need to be included in future studies to provide conclusive results.

\section{ACKNOWLEDGMENTS}

Partially supported by the Research Council of Norway through its Centres of Excellence scheme, project numbers 262762 and 250698. Thanks to the UiO Science Library, and particularly Solveig Isis Sørbø, for assistance in organizing and running the concert.

\section{REFERENCES}

[1] Anne J. Blood and Robert J. Zatorre. 2001. Intensely pleasurable responses to music correlate with activity in brain regions implicated in reward and emotion. Proceedings of the National Academy of Sciences 98, 20 (2001), 11818-11823. https: //doi.org/10.1073/pnas.191355898

[2] K. Brantberg, L. Löfqvist, and P. Fransson. 2004. Large Vestibular Evoked Myogenic Potentials in Response to Bone-Conducted Sounds in Patients with Superior Canal Dehiscence Syndrome. Audiology and Neurotology 9, 3 (2004), 173-182. https://doi.org/10.1159/000077268

[3] Alexandre Coste, Robin N. Salesse, Mathieu Gueugnon, Ludovic Marin, and Benoit G. Bardy. 2017. Standing or swaying to the beat: Discrete auditory rhythms entrain stance and promote postural coordination stability. Gait \& Posture 59 (2017), 28-34. https://doi.org/10.1016/j.gaitpost.2017.09.023

[4] Ariany G. da Silva, Heraldo L. Guida, Ana MÃąrcia dos S. Ant Ãtnio, Renata S. Marcomini, Anne M.G.G. Fontes, Luiz Carlos de Abreu, Adriano L. Roque, Sidney B. Silva, Rodrigo D. Raimundo, Celso Ferreira, and Vitor E. Valenti 2014. An exploration of heart rate response to differing music rhythm and tempos. Complementary Therapies in Clinical Practice 20, 2 (2014), 130-134. https://doi.org/10.1016/j.ctcp.2013.09.004

[5] Marco Donnarumma. 2011. Xth Sense: A study of muscle sounds for an experimental paradigm of musical performance. In Proceedings of the International Computer Music Conference. University of Huddersfield, 243-248.

[6] Rolf Inge Godøy, Minho Song, Kristian Nymoen, Mari Romarheim Haugen, and Alexander Refsum Jensenius. 2016. Exploring Sound-Motion Similarity in Musical Experience. Journal of New Music Research 45, 3 (2016), 210-222. https://doi. org/10.1080/09298215.2016.1184689

[7] Anthony Gritten and Elaine King. 2006. Music and Gesture. Ashgate, Wiltshire.

[8] John Iversen and Aniruddh Patel. 2008. The Beat Alignment Test (BAT): Surveying Beat Processing Abilities in the General Population. In Proceedings of the 10th
International Conference on Music Perception and Cognition (ICMPC10).

[9] Alexander Refsum Jensenius, Agata Zelechowska, and Victor Evaristo Gonzalez Sanchez. 2017. The Musical Influence on People's Micromotion when Standing Still in Groups. In Proceedings of the Sound and Music Computing Conference. Aalto University, 195-200.

[10] Marc Leman. 2016. The expressive moment: how interaction (with music) shapes human empowerment. MIT Press, Cambridge, MA

[11] Micheline Lesaffre, Pieter-Jan Maes, and Marc Leman (Eds.). 2017. The Routledge Companion to Embodied Music Interaction (1 edition ed.). Routledge, New York ; London.

[12] Guy Madison. 2006. Experiencing Groove Induced by Music: Consistency and Phenomenology. Music Perception: An Interdisciplinary fournal 24, 2 (2006), 201-208. http://www.jstor.org/stable/10.1525/mp.2006.24.2.201

[13] Pieter-Jan Maes, Marc Leman, Caroline Palmer, and Marcelo Wanderley. 2014. Action-based effects on music perception. Frontiers in Psychology 4 (2014), 1008. https://doi.org/10.3389/fpsyg.2013.01008

[14] Ernest Mas-Herrero, Josep Marco-Pallares, Urbano Lorenzo-Seva, Robert J. Zatorre, and Antoni Rodriguez-Fornells. 2013. Individual Differences in Music Reward Experiences. Music Perception: An Interdisciplinary fournal 31, 2 (2013), 118-138. https://doi.org/10.1525/mp.2013.31.2.118 arXiv:http://mp.ucpress.edu/content/31/2/118.full.pdf

[15] Dirk Moelants, Michiel Demey, Maarten Grachten, Chia-Fen Wu, and Marc Leman. 2012. The Influence of an Audience on Performers: A Comparison Between Rehearsal and Concert Using Audio, Video and Movement Data. Fournal of New Music Research 41, 3-4 (2012), 67-78. https://doi.org/10.1080/09298215. 2011.642392

[16] Jessica Phillips-Silver and Laurel J. Trainor. 2008. Vestibular influence on auditory metrical interpretation. Brain and Cognition 67, 1 (2008), 94-102. https://doi. org/10.1016/j.bandc.2007.11.007

[17] Kianoush Sheykholeslami, Mohammad Habiby Kermany, and Kimitaka Kaga. 2001. Frequency sensitivity range of the saccule to bone-conducted stimuli measured by vestibular evoked myogenic potentials. Hearing Research 160 (2001), 58-62. https://doi.org/10.1016/S0378-5955(01)00333-1

[18] Yi-Huang Su. 2016. Sensorimotor Synchronization with Different Metrical Levels of Point-Light Dance Movements. Frontiers in Human Neuroscience 10, 186 (2016). https://doi.org/10.3389/fnhum.2016.00186

[19] Neil P. Todd. 1999. Motion in Music: A Neurobiological Perspective. Music Perception: An Interdisciplinary fournal 17, 1 (1999), 115-126. https://doi.org/10. 2307/40285814

[20] Neil P. McAngus Todd and Frederik W. Cody. 2000. Vestibular responses to loud dance music: A physiological basis of the "rock and roll threshold"? The fournal of the Acoustical Society of America 107 (2000), 496-500. https://doi.org/10.1121/ 1.428317

[21] B. J. Yates. 1992. Vestibular influences on the sympathetic nervous system. Brain Research Reviews 17, 1 (1992), 51-59. https://doi.org/10.1016/0165-0173(92) 90006-8 